\title{
Immune-related pneumonitis associated with immune checkpoint inhibitors in lung cancer: a network meta-analysis
}

Xinru Chen (D) , ${ }^{1}$ Zhonghan Zhang, ${ }^{1}$ Xue Hou, ${ }^{1}$ Yaxiong Zhang, ${ }^{1}$ Ting Zhou, ${ }^{1}$ Jiaqing Liu, ${ }^{1}$ Zhihuan Lin, ${ }^{1}$ Wenfeng Fang, ${ }^{1}$ Yunpeng Yang, ${ }^{1}$ Yuxiang $\mathrm{Ma},{ }^{2}$ Yan Huang, ${ }^{1}$ Hongyun Zhao, ${ }^{2}$ Li Zhang ${ }^{1}$
To cite: Chen $X$, Zhang $Z$, Hou X, et al. Immune-related pneumonitis associated with immune checkpoint inhibitors in lung cancer: a network meta-analysis. Journal for ImmunoTherapy of Cancer 2020;8:e001170. doi:10.1136/ jitc-2020-001170

$\mathrm{XC}, \mathrm{ZZ}$ and XH contributed equally.

Accepted 24 July 2020

\section{Check for updates}

(c) Author(s) (or their employer(s)) 2020. Re-use permitted under CC BY-NC. No commercial re-use. See rights and permissions. Published by BMJ.

${ }^{1}$ Department of Medical Oncology, Sun Yat-sen University Cancer Center, State Key Laboratory of Oncology in South China, Collaborative Innovation Center for Cancer Medicine, Sun Yat-sen University Cancer Center, Guangzhou, Guangdong, China

${ }^{2}$ Department of Clinical

Research, Sun Yat-sen University Cancer Center, State Key Laboratory of Oncology in South China, Collaborative Innovation Center for Cancer Medicine,

Sun Yat-sen University Cancer Center, Guangzhou, China

\section{Correspondence to}

Dr Li Zhang;

zhangli6@mail.sysu.edu.cn

Dr Hongyun Zhao;

zhaohy@sysucc.org.cn

\section{ABSTRACT}

Background Immune checkpoint inhibitors (ICls) have dramatically revolutionized lung cancer treatment, providing unprecedented clinical benefits. However, immune-related pneumonitis (IRP) caused by ICls has aroused widespread concern due to its high rate of discontinuation and mortality. This network meta-analysis (NMA) aims to compare the risks of IRP among different regimens for advanced lung cancer.

Methods Phase II and III randomized clinical trials (RCTs) were searched from electronic databases. The rates of grade 1-5 IRP and grade 3-5 IRP were systematically extracted. An NMA was conducted among chemotherapy, ICls monotherapy, dual ICls combination, and

ICls+chemotherapy. Subgroup analysis was also compared based on specific types of ICls.

Results Twenty-five RCTs involving 17,310 patients were eligible for inclusion. Compared with chemotherapy, ICl-based regimens were associated with an increased risk of grade 1-5 IRP and grade 3-5 IRP. Compared with ICls+chemotherapy, ICls monotherapy (grade 1-5: OR $2.14,95 \%$ credible interval 1.12 to 4.80 ; grade $3-5$ : 3.03 , 1.491 to 6.69 ) and dual ICls combination (grade 1-5: 3.86, 1.69 to 9.89; grade 3-5: 5.12, 2.01 to 13.68) were associated with a higher risk of grade 1-5 IRP and grade 3-5 IRP. No significant difference was found between dual ICls combination and ICls monotherapy in grade 1-5 $\operatorname{IRP}(1.85,0.91$ to 3.37$)$ or in grade $3-5 \operatorname{IRP}(1.65,0.81$ to 3.37$)$. Besides, compared with programmed cell death protein 1 (PD-1) inhibitors (2.56, 1.12 to 6.60$)$, a lower risk of grade 1-5 IRP was observed in programmed cell death ligand 1 (PD-L1) inhibitors.

Conclusion Compared with chemotherapy, using ICls is associated with an increased risk of IRP. $\mathrm{ICls+chemotherapy} \mathrm{is} \mathrm{associated} \mathrm{with} \mathrm{a} \mathrm{lower} \mathrm{risk}$ of IRP compared with dual ICls combination and ICls monotherapy. PD-1 inhibitors are associated with a higher risk of 1-5 grade IRP compared with PD-L1 inhibitors.

\section{INTRODUCTION}

Chemotherapy alone is gradually falling out of favor in the treatment of advanced lung cancer due to the rapid development of immune checkpoint inhibitors (ICIs). In recent years, ICIs alone, as well as in a combination regimen have succeeded in changing the treatment landscape of advanced lung cancer, with longer survival made possible by their emergence. ${ }^{1} 2$ With dramatically increased drug availability, a large proportion of patients now receive ICIs. However, immune-related adverse effects (irAEs) commonly emerge as side effects which exist on a uniquely variable spectrum. One particular worrying irAE is the development of immune-related pneumonitis (IRP), which is more common in non-small-cell lung cancer (NSCLC). ${ }^{34}$ One possible reason may be related to patients with lung cancer and the high number of those that smoke, along with those with underlying pulmonary conditions such as chronic obstructive pulmonary disease (COPD), and those who have undergone prior thoracic radiation. ${ }^{5-7}$

IRP is an inflammatory infiltrative lung disease associated with ICIs that results in a high rate of treatment discontinuation and mortality in patients with lung cancer thus arousing great concerns among clinicians. ${ }^{89}$ Previous pairwise meta-analyses have reported the incidence of IRP in NSCLC was between $3.6 \%$ and $4.1 \%$, and that it also has a higher rate of IRP with the use of programmed cell death protein 1 (PD-1) inhibitors compared with programmed cell death ligand 1 (PD-L1) inhibitors. ${ }^{3} 1011$ Recently, with increasingly diverse therapeutic options for advanced lung cancer, ICIs+chemotherapy, or dual ICIs combination have become the standard firstline treatment regimens. ${ }^{12}$ Yet, whether these combination regimens increase the incidence of IRP remains unknown.

What is known, is that pairwise metaanalyses only make comparisons between two treatment arms, thus failing to cover all possible treatment regimen comparisons. Whereas, a network meta-analysis (NMA) has the advantage of comparing multiple treatments simultaneously by combining direct 
and indirect evidence, and enables the ranking of interventions. Therefore, we conducted an NMA with all the available up-to-date ICI-related lung cancer patients to compare the risk of IRP in regards to various regimens.

\section{METHODS}

\section{Data sources and searches}

PubMed, Embase and the Cochrane Library were searched for relevant articles up to April 20, 2020 in the English languages using a combination of the following main research terms 'lung cancer' and 'checkpoint inhibitors' with the searches restricted to 'randomized controlled trials (RCTs)' only. The search strategy is as described in online supplementary table S1.

Next, we searched for unpublished data at 'ClinicalTrials.gov'. In order to include as many available results as possible, we extracted data from the results part of ClinicalTrials.gov and reviewed the abstracts and presentations on lung cancer from the recent oncology congress ASCO (American Society of Clinical Oncology); ESMO (the European Society for Medical Oncology); ESMO-Asia; and the WCLC (World Conference on Lung Cancer) up to December 30, 2019. The detailed search terms are listed in online supplementary table S2.

\section{Selection criteria}

This study compared the risk of IRP among all kinds of ICI-based regimens and chemotherapy for patients with advanced lung cancer. The inclusion criteria included the following: head-to-head phase II and phase III RCTs which had enrolled patient with pathologically confirmed advanced lung cancer, patients should have received ICI treatment (on at least one treatment arm), and trials must have reported the incidence of IRP and its grading. Trials not adhering to the above criteria were excluded.

\section{Data extraction and risk of bias assessment}

Two authors (XC and ZZ) reviewed all trials from the search results and extracted the following data independently: trial name, National Clinical Trial number, publication year, tumor types, study phase, treatment drugs, line of therapy, sample size, age, gender, smoking status, performance status (PS), prior radiotherapy, and incidence of 1-5 grade and grade 3-5 IRP. Other related pulmonary diseases listed as pneumonia or interstitial lung disease were not extracted.

The Cochrane Risk of Bias Tool was used to assess the risk of bias for each RCT. ${ }^{13}$ The quality of the studies were assessed independently by our two reviewers (XC and $\mathrm{XH})$ and a consensus reached following any concerns.

\section{Data synthesis and statistical analyses}

First, we generated loops to illustrate the network geometry using Stata V.13.0 to depict which treatments were compared directly or indirectly. Second, pair-wise metaanalyses on head-to-head comparisons by using Review Manager V.5.3 (Cochrane Collaboration) were carried out to make direct estimates. Pooled ORs with the corresponding $95 \%$ CI was used to present the results. The heterogeneity of the included studies were evaluated by $\mathrm{I}^{2}$ statistics and $\mathrm{p}$ value within a visual forest plot. Values of $\mathrm{I}^{2}$ under $25 \%$, between $25 \%$ and $50 \%$, and over $50 \%$ were regarded as low, moderate, and high heterogeneity, respectively. ${ }^{14}$ A random effects model was used if heterogeneity existed; otherwise, the fixed effects model was applied. Then, a random-effects model within a Bayesian framework was established using the Markov chain Monte Carlo methods in Aggregate Data Drug Information System 1.16.8 (Drugis.org). ${ }^{15}$ The Brooks-Gelman-Rubin method was applied to assess the model's degree of convergence. This method compared chain and between-chain variance to calculate the potential scale reduction factor (PSRF). ${ }^{16}{ }^{17}$ The more PSRF approximate to 1 , the better convergence was obtained, and a PSRF $\leq 1.05$ was deemed acceptable. More detailed parameters are presented in online supplementary tables S3 and S4. The results are reported as ORs and CI. If there were no inconsistencies in the evidence, a consistency model was used to conclude the relative effect of the included treatments; otherwise, an inconsistency model would be applied.

Inconsistency appraisal was achieved via two steps. First, we made a general comparison between the consistency model and the inconsistency model, calculating for inconsistency factors (IF), inconsistency standard deviation (ISD) and random effects standard deviation (RESD). If the 95\% CI of IF contained ' 0 ' and the $95 \%$ CI of ISD included ' 1 ', and the RESD between the consistency model and the inconsistency model was roughly equal; it was considered to have strong data consistency. Second, node-splitting models were adopted to identify any inconsistencies, significant inconsistency was defined as a $p$ value less than $(<) 0.05$. The probability of treatment ranking was based on the surface under the cumulative ranking curve (SUCRA), and higher SUCRA scores correlated with a higher risk of IRP.

Transitivity assumption was evaluated by comparing the distribution of potential modified treatment effects (effect modifiers) across treatment comparisons. ${ }^{18}$ Since all included studies were those that enrolled patients with advanced lung cancer, and all studies were RCTs without significant methodological heterogeneity, the baseline parameters were the crucial factors in determining transitivity.

Sensitivity analyses were performed to assess the robustness of each study by restricting the following factors: phase III RCTs, published studies, studies that included ICI-based first-line therapy only, and studies that enrolled patients with NSCLC. Owing to the low number of patients who were treated with second and later line treatments, the sensitivity analysis was not conducted in this subgroup of patients.

A 'comparison-adjusted' funnel plot was used to assess publication bias (eg, small-study effects) within a network of interventions. 
PubMed/Embase/Cochrane Library

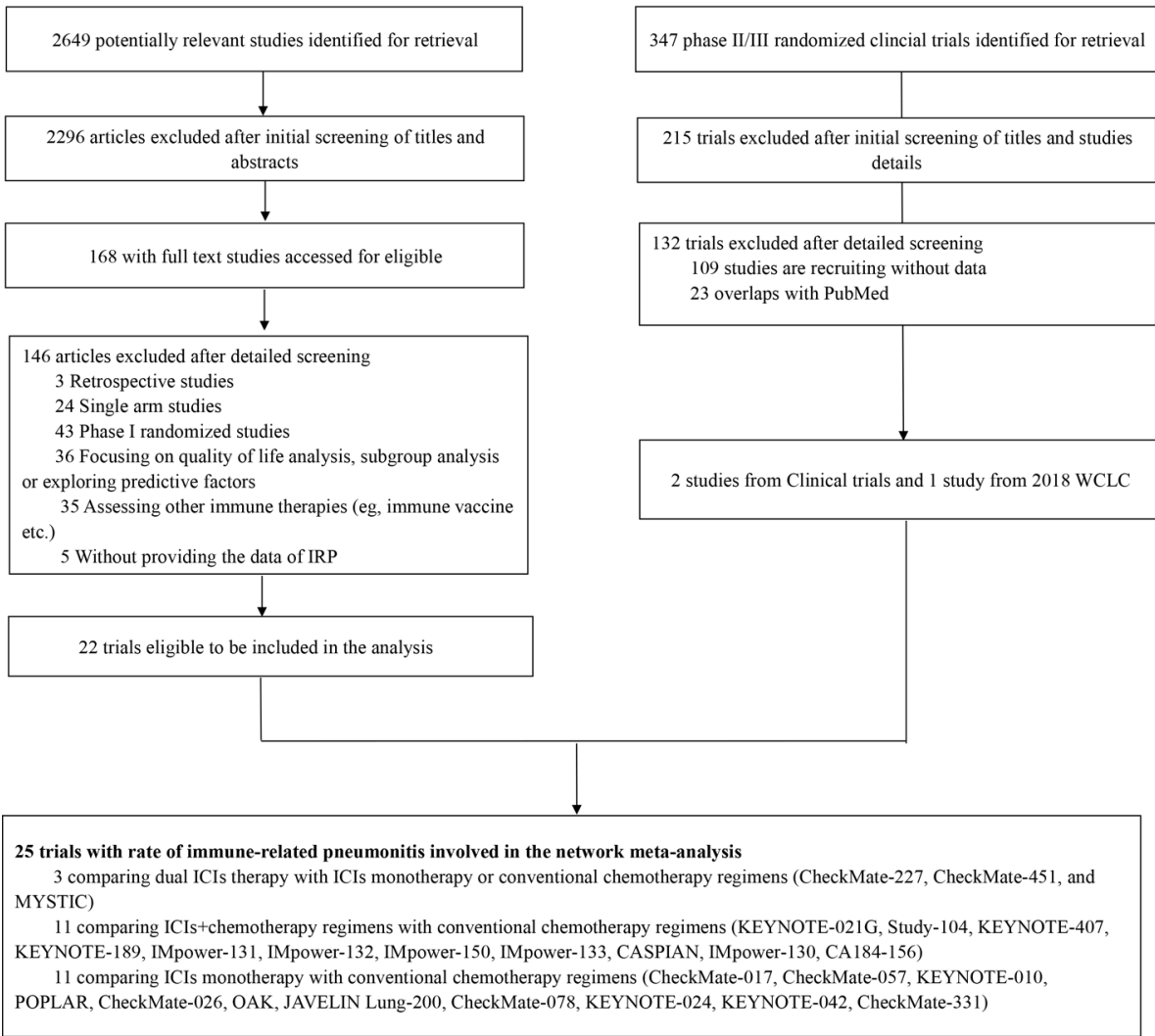

Figure 1 Flowchart of study selection. ICls, immune checkpoint inhibitors; IRP, immune-related pneumonitis; WCLC, World Conference on Lung Cancer.

\section{RESULTS}

\section{Study selection}

A total of 168 studies with full-text articles accessed for eligibility from the three electronic databases, and 22 RCTs $^{19-33}$ with 15,641 patients meeting this NMA's inclusion criteria (figure 1). There were 132 RCTs involving ICIs retrieved from 'ClinicalTrials.gov'. After reviewing their characteristics, three RCTs were eligible for inclusion with 1669 patients included in this NMA (figure 1). The incidence of IRP from two trials (CheckMate-331, CheckMate-451) were extracted from ClinicalTrials.gov and one trial from the 2018 WCLC meeting abstract (IMpower132). In total, 25 randomized trials that contained the occurrence rate of IRP were included in this study.

The Cochrane tool for risk of bias was used to measure the quality of each study. All 25 studies were evaluated and random sequence generation performed. One study displayed an unclear risk of bias in regards to allocation concealment. With respect to performance bias, 19 studies were conducted as open-label with a high risk of performance bias and only six studies with double-blind design. Two studies had incomplete outcome data due to a lack of 3-5 IRP data. Three unpublished studies had an unclear risk of reporting bias, and other bias. The detailed assessment results were shown in online supplementary figure S1. No obvious publication bias was observed in this NMA; the funnel plots were roughly symmetrical and near the zero line (online supplementary figures S2 and S3).

\section{Study characteristic and treatment group description}

Online supplementary table S5 summarizes the main characteristics of all the trials included in this NMA. There were 22 phase III trials, 2 phase II trials, and one phase II/III trial. Twenty studies had data from patients with NSCLC and five studies from patients with smallcell lung cancer (SCLC). There were 32 two arms RCTs, with two studies having three arms; the most common treatment arm was chemotherapy $(n=24,96.0 \%)$. The incidence of grade 1-5 IRP and grade 3-5 IRP was available in almost all RCTs, except for two studies (CheckMate-331, CheckMate-451) from ClinicalTrials.gov without the data for grade 3-5 IRP provided. The median follow-up time was 14.8 months (ranging from 7.8 to 42.6 months). The study sample size ranged from 123 to 1271 patients. The median age was 64 years (ranging from 18 to 90 years old), $32.8 \%$ were female, $85.9 \%$ had a history of smoking, and $97.6 \%$ with a PS score $0-1$. Information on the history of prior radiotherapy was not reported in $60 \%$ of the studies $(n=15)$. Across comparisons, the distribution of baseline characteristics by treatment was generally balanced (online supplementary tables S6 and S7). Therefore, we accepted the assumption of transitivity for our NMA. 


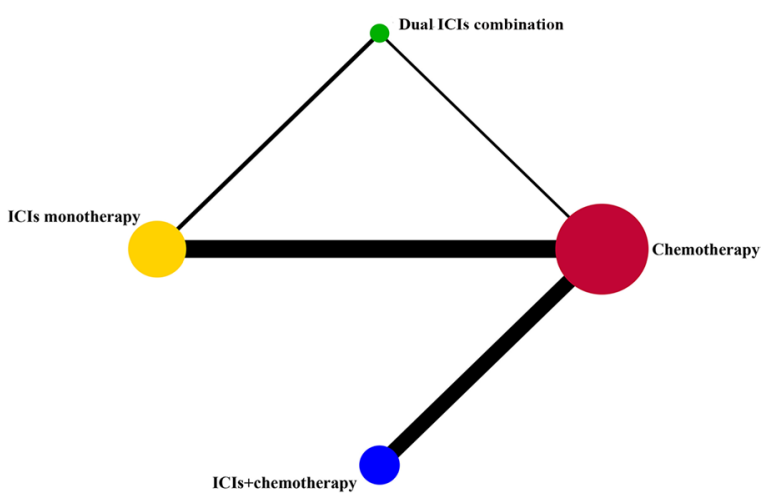

Figure 2 Network established for comparisons based on four treatment groups. Each circular node represents a type of treatment. The node size is proportional to the total number of patients receiving a treatment (in brackets). Each line represents a type of head-to-head comparison. The width of the lines is proportional to the number of trials comparing the connected treatments. ICls, immune checkpoint inhibitors.

The rate of grade 1-5 IRP in different treatment regimens was compared among four groups including chemotherapy, ICIs monotherapy, dual ICIs combination and ICIs+chemotherapy. Given that bevacizumab has little impact on the occurrence of IRP, ${ }^{34} 35$ only one study (IMpower-150) in the ICIs+chemotherapy group added bevacizumab to both the experimental and control arm was included in the NMA. Furthermore, we subdivided the four treatment groups into seven subgroups based on the different types of ICIs: chemotherapy, PD-1 monotherapy, PD-L1 monotherapy, PD-1/PD-L1+cytotoxic T lymphocyte-associated antigen 4 (CTLA-4), PD-1+chemotherapy, PD-L1+chemotherapy, CTLA-4+chemotherapy. Considering the relatively small sample size of PD-1+CTLA-4 and PD-L1+CTLA-4, they were combined into one group. Networks for multiple treatment groups are presented in figure 2 and online supplementary figure S4.

\section{NMA for IRP based on four treatment groups}

According to the established NMA based on the consistency model, conventional chemotherapy had the lowest risk of grade 1-5 IRP compared with ICIs monotherapy (OR, $0.16,95 \%$ CI, 0.09 to 0.25 ), dual ICIs combination $(0.09,0.04$ to 0.18$)$ and ICIs+chemotherapy $(0.34,0.21$ to 0.59 ; table 1$)$. Interestingly, ICIs monotherapy (2.14, 1.12 to 4.80$)$ and dual ICIs combination $(3.86,1.69$ to 9.89) showed a noticeably higher risk of grade 1-5 IRP compared with ICIs+chemotherapy. For grade 3-5 IRP, a total of 23 RCTs were enrolled in the NMA based on the consistency model. The results showed that chemotherapy was associated with a lower risk of IRP compared with ICIs monotherapy $(0.20,0.12$ to 0.33$)$ and dual ICIs combination $(0.12,0.06$ to 0.25$)$, while no significant difference was found in the ICIs+chemotherapy group (0.62, 0.35 to 1.06 ; table 2 ). In line with grade $1-5$ IRP, ICIs monotherapy $(3.03,1.49$ to 6.69$)$ and dual ICIs combination (5.12, 2.01 to 13.68$)$ also presented a higher risk of grade 3-5 IRP than ICIs+chemotherapy. However, a comparable result was observed between ICIs monotherapy and dual ICIs combination in grade 1-5 IRP $(1.85,0.91$ to $3.37)$, and in grade $3-5$ IRP $(1.65,0.81$ to 3.37$)$. SUCRA provided a ranking of each treatment group according to its incidence of IRP. The ranking probability based on the four treatment groups is shown in figure 3 and online supplementary table S8. For the grade 1-5 IRP, it is notable that dual ICIs combination had the highest ranking (0.99) followed by ICIs monotherapy (0.68), ICIs+chemotherapy (0.34) and chemotherapy (0). For grade 3-5 IRP, the ranking was consistent with grade 1-5 IRP from high to low: dual ICIs combination $(0.97)$, ICIs monotherapy (0.69), ICIs+chemotherapy (0.31) and chemotherapy (0.02).

\section{NMA for IRP by different ICIs based on seven treatment groups}

Online supplementary table S9 for grade 1-5 IRP in seven treatment groups based on the consistency model showed that chemotherapy had the lowest incidence of IRP compared with the other six treatment groups without being influenced by the type of ICIs. Of note, compared with PD-L1 inhibitors, a higher risk of grade 1-5 IRP was observed in PD-1 inhibitors. The corresponding ranking of these seven groups from high to low was: PD-1/PD-L1+CTLA-4 (0.96), PD-1 (0.86), PD-L1+chemotherapy (0.50), PD-L1 (0.48), PD-1+chemotherapy (0.47), CTLA-4+chemotherapy (0.15) and chemotherapy (0.09) (online supplementary table S10). In terms of grade $3-5$ IRP, based on the consistency model, less difference was found among treatment groups (online supplementary

Table 1 Multiple treatment comparison for IRP based on network consistency model. (OR $>1$ means the treatment in top left is worse)

\section{OR with $95 \% \mathrm{Cl}$ for grade 1-5 IRP}

\section{Chemotherapy}

Dual ICls combination

$0.34(0.21$ to $\underline{0.59})$
1.85 (0.91 to 3.37 )

3.86 (1.69 to $\underline{9.89)}$
ICls monotherapy

2.14 (1.12 to $\underline{4.80})$

Bold values denote statistical significance.

ICls, immune checkpoint inhibitors; IRP, immune-related pneumonitis. 
Table 2 Multiple treatment comparison for IRP based on network consistency model. (OR $>1$ means the treatment in top left is worse)

\section{OR with $95 \% \mathrm{Cl}$ for grade 3-5 IRP}

\section{Chemotherapy}

$0.12(0.06$ to $\underline{0.25)}$

0.20 (0.12 to $\underline{0.33)}$

0.62 (0.35 to 1.06)
Dual ICls combination

1.65 (0.81 to 3.37$)$

$5.12(2.01$ to 13.68$)$
ICls monotherapy

3.03 (1.49 to $\underline{6.69)}$
ICls+chemotherapy

Bold values denote statistical significance

ICls, immune checkpoint inhibitors; IRP, immune-related pneumonitis.

table S11). There was no significant difference between PD-1 inhibitors and PD-L1 inhibitors. The ranking from high to low was: PD-1/PD-L1+CTLA-4 (0.94), PD-1 (0.85), PD-L1 (0.60), PD-1+chemotherapy (0.47), PD-L1+chemotherapy (0.36), chemotherapy (0.16) and CTLA-4+chemotherapy (0.13) (online supplementary table S10).

\section{Heterogeneity and inconsistency assessment}

Four feasible pairwise comparisons with heterogeneity estimates are presented in online supplementary figures S5 and S6. Three comparisons were (ICIs monotherapy, dual ICIs combination and ICIs+chemotherapy) versus chemotherapy. One comparison was ICIs monotherapy versus dual ICIs combination. Almost all comparisons suggested a low heterogeneity either in grade 1-5 IRP or grade 3-5 IRP, and only one comparison (ICIs monotherapy vs dual ICIs combination in grade 3-5 IRP) showed high heterogeneity. The results of these four comparisons also demonstrated remarkable consistency in tendency in relation to the corresponding NMA results. The results of the inconsistency evaluation are presented in online supplemental tables 12-15. Both the consistence models fitted well with the inconsistency model. The node splitting analyses also showed no significant inconsistency.

\section{Sensitivity analysis}

There were 22 phase III RCTs, 22 published studies, of which 16 studies included ICI-based first-line therapy only, and 20 studies that enrolled patients with NSCLC

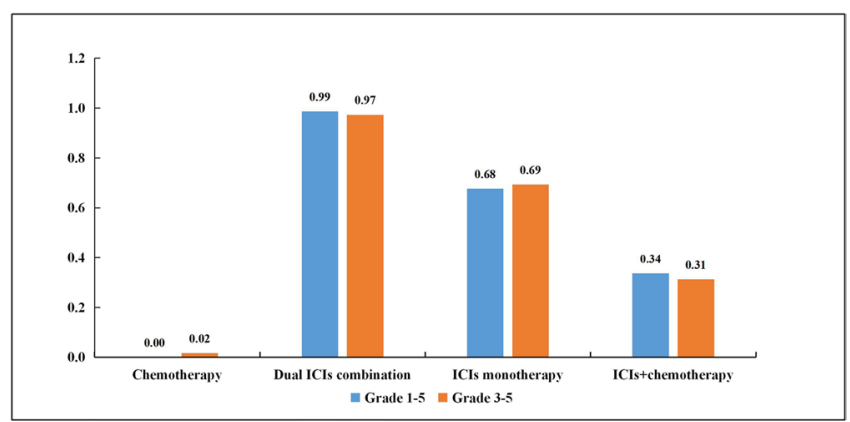

Figure 3 Rank probabilities with SUCRA value for immunerelated pneumonitis (IRP) in four treatment groups based on the network consistency model. Higher SUCRA scores are correlated with higher risk of IRP. ICls, immune checkpoint inhibitors; SUCRA, surface under the cumulative ranking curve. were included into the sensitivity analyses separately. The ranking order of grade 1-5 IRP and grade 3-5 IRP in the four treatment groups showed remarkable consistency with the original NMA (online supplementary table S16). With respect to seven treatment groups, only the ranking order of grade 1-5 IRP in 16 studies that included patients who received ICI-based first-line treatment showed a slight difference in which PD-1 inhibitors were higher than that of a PD-1/PD-L1+CTLA-4 combination (online supplementary table $\mathrm{S} 17$ ).

\section{DISCUSSION}

ICIs have emerged as one of the most significant treatment choices for advanced lung cancer, however, their expanded use comes with noticeable growth in IRP. ${ }^{32} 36$ Past binary meta-analyses have demonstrated that IRP incidence was higher with ICIs combination immunotherapy versus monotherapy and might be different among various types of ICIs. ${ }^{37-39}$ Besides, few sparse NMA have evaluated the risk of irAEs among different ICIs. Moreover all these studies were not IRP specific and limited by small trails sizes necessitating the pooling of different tumor types and types of irAEs. ${ }^{40-42}$ With more treatment options now approved for advanced lung cancer, a robust NMA is urgently needed to comprehensively analyze and compare the risk of IRP for all kinds of treatment regimens. This current NMA included 25 head-to-head phase II and III RCTs (17,310 patients), and to our knowledge, is the first and the latest NMA to compare the risk of IRP among ICI-based regimens and chemotherapy for advanced lung cancer. In particular, current studies on extensive SCLC were also included in our NMA.

Our study showed that ICI-based regimens were associated with a higher risk of grade $1-5$ and grade $3-5$ IRP compared with conventional chemotherapy. Consistent with a recent binary meta-analysis carried out by Magee and colleagues, the results showed that patients with lung cancer had more cases of pneumonitis compared with other solid-organ malignancies, and exposure to immunotherapy increased the risk of pneumonitis compared with chemotherapy. ${ }^{43}$ Interestingly, another unique finding we observed was that ICI+chemotherapy decreased the risk of grade 1-5 IRP compared with ICIs monotherapy and dual ICIs combination. This trend was 
also detected in the grade 3-5 IRP. Contrary to our study, one NMA concerning multiple cancer types reported by $\mathrm{Xu}$ and colleagues found that the risk of IRP in ICIs with conventional therapies ranked first, followed by dual ICIs combination and ICIs monotherapy. ${ }^{44}$ The reason for this, might be explained by the types of tumors included in such NMA, only 12 out of 26 trials were NSCLC, and most of the studies had not been published at that time (by the end of February 2018). Our NMA focus on lung cancer and a population group susceptible to IRP, which may be helpful for clinicians in selecting appropriate ICI regimens. Though we cannot neglect other irAEs when ICI drugs are being prescribed. One possible reason for the decreased risk of IRP when chemotherapy is used in combination with ICIs may lie in the fact that conventional chemotherapy consists of cytotoxic agents that are believed to cause chemotherapy-induced immunosuppression; adding further stress on the whole immune system and resulting in lower responding immune function. ${ }^{45}{ }^{46}$ Another important factor that may be involved in the decreased risk of IRP is the use of corticosteroids, as binding pretreatment is commonly used for antiemetic and antiallergy purpose in chemotherapy regimens containing platinum, pemetrexed and taxanes. Also, it is well known that corticosteroids possess the ability to suppress the immune system ${ }^{47} 48$ and play a significant role in the therapy of certain underlying pulmonary conditions like asthma and COPD, radiation pneumonitis caused by thoracic radiation and even in IRP treatment according to various guidelines. ${ }^{49}{ }^{50}$ Unfortunately, how cytotoxic agents and corticosteroids precisely suppress and regulate the immune system remains unclear and needs further exploration. More detailed basic research and prospective RCTs are still warranted to address this important clinical issue.

In line with the results from several binary meta-analyses, our large-scale NMA further confirmed that PD-L1 inhibitors were safer than PD-1 inhibitors with respect to the risk of IRP, which may help clinicians when choosing different types of ICIs. A possible reason for a higher risk when using PD-1 inhibitors might be explained by research from Xiao and colleagues which demonstrates that PD-1 inhibitors themselves may have the ability to change the balance in PD-L2 interaction with repulsive guidance molecule b, which could lead to pneumonitis; however, PD-L1 inhibitors are unable to disrupt this shift. ${ }^{51}$ But we should note that a comparable result of grade 3-5 IRP was found between PD-1 inhibitors and PD-L1 inhibitors. This might indicate that for severe IRP, and even for some fatal IRP, the pulmonary toxicity of the PD- 1 inhibitors does not really outweigh the use of PD-L1 inhibitors.

It should be noted that some studies have pointed out that the incidence of IRP when using dual ICIs combination was higher than in ICIs monotherapy. ${ }^{337}$ In our study, no statistical differences were observed between these two groups; however, this trend can be seen from the ranking either in grade 1-5 IRP or grade 3-5 IRP. Given the fact that the number of studies directly compared dual ICIs combination and ICIs monotherapy was limited, we need to interpret these results with caution. More high-quality RCTs are needed to investigate the incidence of IRP between ICIs monotherapy and dual ICIs combination.

The present study has several limitations. One, the reporting incidence of IRP may have increased incrementally over time as the number of patients receiving ICIs increased. A large proportion of studies in our NMA were continuously updated. Two, many RCTs enrolled in our NMA were open-label and only $24 \%$ of trials were assessed blindly. Besides, in the data extraction process, our study only included patients that were clearly noted as pneumonitis and excluded those listed as 'pneumonia' and 'interstitial lung disease'. However, a consensus diagnostic criteria of IRP is still lacking, and some clinical symptoms, such as a cough, dyspnea, low oxygen saturation, and fever, are non-specific. In clinical trials, a large proportion of patients diagnosed with IRP were based on the experience of each clinician and were not centrally reviewed. Therefore, the identification of IRP might not be completely accurate and might lead to bias for the evaluation of IRP. Three, the small sample sizes in some of the treatment arms such as dual ICIs combination versus ICIs monotherapy. Additionally, patients were not stratified according to a larger variety of checkpoint inhibitors and chemotherapy drugs because of the limited sample sizes. Ideally, the analysis should differentiate between the classes of ICIs, for which some meta-analyses have come up with conclusions that different ICIs might have distinctive toxicity spectrums. Four, high-risk factors for IRP, such as a history of prior RT and smoking status, were not controlled for when our NMA was carried out. Finally, we only enrolled trials reported in English, given practical constraints with the translations of reports in other languages. Despite these limitations we found meaningful results which may help clinicians choose suitable ICI-based regimens. Soon, we expect more double-blind RCTs highlighting head-to-head comparisons focusing on various ICIs or combination treatments and results from real-world studies are urgently needed to confirm our findings.

\section{CONCLUSION}

ICI-based regimens have a higher risk of IRP than chemotherapy in general. In terms of ICI-based regimens, ICIs+chemotherapy is associated with a lower risk of IRP than ICIs monotherapy and dual ICIs combination therapy. Moreover, subgroup analysis also showed that PD-1 inhibitors are associated with a higher risk of 1-5 grade IRP compared with PD-L1 inhibitors. These findings may be valuable in clinical decision-making in the absence of hard evidence from RCTs.

Contributors Conception/design: XC, ZZ, HZ and LZ. Collection and/or assembly of data: XC, ZZ, XH, YZ, TZ, JL. Data analysis and interpretation: XC, ZZ, XH, YZ, TZ, JL, Linzhi Huan, WF, YY, YM, YH, HZ, LZ. Manuscript writing: ZZ, YZ, XH, HZ, LZ. Final approval of manuscript: XC, ZZ, XH, YZ, TZ, JL, ZL, WF, YY, YM, YH, HZ, LZ. 
Funding This work was supported by Medical Scientific Research Foundation of Guangdong Province, China (Grant No. A2020153).

Competing interests None declared.

Patient consent for publication Not required.

Provenance and peer review Not commissioned; externally peer reviewed.

Data availability statement Data are available in a public, open access repository. All data relevant to the study are included in the article or uploaded as supplementary information. The data used to support the findings of this study are included within the manuscript and supplementary files.

Open access This is an open access article distributed in accordance with the Creative Commons Attribution Non Commercial (CC BY-NC 4.0) license, which permits others to distribute, remix, adapt, build upon this work non-commercially, and license their derivative works on different terms, provided the original work is properly cited, appropriate credit is given, any changes made indicated, and the use is non-commercial. See http://creativecommons.org/licenses/by-nc/4.0/.

ORCID iD

Xinru Chen http://orcid.org/0000-0002-9093-1911

\section{REFERENCES}

1 Remon J, Passiglia F, Ahn M-J, et al. Immune checkpoint inhibitors in thoracic malignancies: review of the existing evidence by an IASLC expert panel and recommendations. J Thorac Oncol 2020;15:914-47.

2 lams WT, Porter J, Horn L. Immunotherapeutic approaches for smallcell lung cancer. Nat Rev Clin Oncol 2020;17:300-12.

3 Nishino M, Giobbie-Hurder A, Hatabu H, et al. Incidence of programmed cell death 1 Inhibitor-Related pneumonitis in patients with advanced cancer: a systematic review and meta-analysis. JAMA Oncol 2016;2:1606-16.

4 Nishino M, Ramaiya NH, Awad MM, et al. Pd-1 Inhibitor-Related pneumonitis in advanced cancer patients: radiographic patterns and clinical course. Clin Cancer Res 2016;22:6051-60.

5 Coussens LM, Werb Z. Inflammation and cancer. Nature 2002;420:860-7

6 Bouros D, Hatzakis K, Labrakis $\mathrm{H}$, et al. Association of malignancy with diseases causing interstitial pulmonary changes. Chest 2002;121:1278-89.

7 Shaverdian N, Lisberg AE, Bornazyan K, et al. Previous radiotherapy and the clinical activity and toxicity of pembrolizumab in the treatment of non-small-cell lung cancer: a secondary analysis of the KEYNOTE-001 phase 1 trial. Lancet Oncol 2017;18:895-903.

8 Disayabutr S, Calfee CS, Collard HR, et al. Interstitial lung diseases in the hospitalized patient. BMC Med 2015;13.

9 Naidoo J, Page DB, Li BT, et al. Toxicities of the anti-PD-1 and anti-PD-L1 immune checkpoint antibodies. Ann Oncol 2016;27:1362.

10 Khunger M, Rakshit S, Pasupuleti V, et al. Incidence of Pneumonitis With Use of Programmed Death 1 and Programmed Death-Ligand 1 Inhibitors in Non-Small Cell Lung Cancer: A Systematic Review and Meta-Analysis of Trials. Chest 2017;152:271-81.

11 Sun X, Roudi R, Dai T, et al. Immune-Related adverse events associated with programmed cell death protein-1 and programmed cell death ligand 1 inhibitors for non-small cell lung cancer: a PRISMA systematic review and meta-analysis. BMC Cancer 2019;19:558.

12 Ettinger DS, Wood DE, Aggarwal C, et al. NCCN guidelines insights: non-small cell lung cancer, version 1.2020. J Natl Compr Canc Netw 2019;17:1464-72.

13 Higgins JPT, Altman DG, Gøtzsche PC, et al. The Cochrane collaboration's tool for assessing risk of bias in randomised trials. BMJ 2011;343:d5928.

14 Bowden J, Tierney JF, Copas AJ, et al. Quantifying, displaying and accounting for heterogeneity in the meta-analysis of RCTs using standard and generalised Q statistics. BMC Med Res Methodol 2011;11:41.

15 Salanti G, Higgins JPT, Ades AE, et al. Evaluation of networks of randomized trials. Stat Methods Med Res 2008;17:279-301.

16 Brooks SP, Gelman A. General methods for monitoring convergence of iterative simulations. Journal of Computational and Graphical Statistics 1998;7:434-55.

17 van Valkenhoef G, Lu G, de Brock B, et al. Automating network meta-analysis. Res Synth Methods 2012;3:285-99.
18 Higgins JPT, Jackson D, Barrett JK, et al. Consistency and inconsistency in network meta-analysis: concepts and models for multi-arm studies. Res Synth Methods 2012;3:98-110.

19 Govindan R, Szczesna A, Ahn M-J, et al. Phase III trial of ipilimumab combined with paclitaxel and carboplatin in advanced squamous non-small-cell lung cancer. $J$ Clin Oncol 2017;35:3449-57.

20 Fehrenbacher L, Spira A, Ballinger M, et al. Atezolizumab versus docetaxel for patients with previously treated non-small-cell lung cancer (poplar): a multicentre, open-label, phase 2 randomised controlled trial. Lancet 2016;387:1837-46.

21 Fehrenbacher L, von Pawel J, Park K, et al. Updated efficacy analysis including secondary population results for oak: a randomized phase III study of Atezolizumab versus docetaxel in patients with previously treated advanced non-small cell lung cancer. J Thorac Oncol 2018;13:1156-70.

22 Rizvi NA, Cho BC, Reinmuth N, et al. Durvalumab with or without tremelimumab vs standard chemotherapy in first-line treatment of metastatic non-small cell lung cancer: the MYSTIC phase 3 randomized clinical trial. JAMA Oncol 2020;6:661-74.

23 Paz-Ares L, Luft A, Vicente D, et al. Pembrolizumab plus chemotherapy for squamous Non-Small-Cell lung cancer. $N$ Engl J Med Overseas Ed 2018;379:2040-51.

24 Gandhi L, Rodríguez-Abreu D, Gadgeel S, et al. Pembrolizumab plus chemotherapy in metastatic Non-Small-Cell lung cancer. N Engl J Med Overseas Ed 2018;378:2078-92.

25 Mok TSK, Wu Y-L, Kudaba I, et al. Pembrolizumab versus chemotherapy for previously untreated, PD-L1-expressing, locally advanced or metastatic non-small-cell lung cancer (KEYNOTE-042): a randomised, open-label, controlled, phase 3 trial. Lancet 2019;393:1819-30.

26 Reck M, Rodríguez-Abreu D, Robinson AG, et al. Updated Analysis of KEYNOTE-024: Pembrolizumab Versus Platinum-Based Chemotherapy for Advanced Non-Small-Cell Lung Cancer With PD-L1 Tumor Proportion Score of $50 \%$ or Greater. J Clin Oncol 2019;37:537-46

27 Socinski MA, Jotte RM, Cappuzzo F, et al. Atezolizumab for firstline treatment of metastatic Nonsquamous NSCLC. N Engl J Med 2018;378:2288-301.

28 Horn L, Mansfield AS, Szczęsna A, et al. First-Line Atezolizumab plus chemotherapy in extensive-stage small-cell lung cancer. $N$ Engl J Med 2018;379:2220-9.

29 Jotte R, Cappuzzo F, Vynnychenko I, et al. Atezolizumab in Combination With Carboplatin and Nab-Paclitaxel in Advanced Squamous NSCLC (IMpower131): Results From a Randomized Phase III Trial. J Thorac Oncol 2020;15:1351-60.

30 West H, McCleod M, Hussein M, et al. Atezolizumab in combination with carboplatin plus nab-paclitaxel chemotherapy compared with chemotherapy alone as first-line treatment for metastatic non-squamous non-small-cell lung cancer (IMpower130): a multicentre, randomised, open-label, phase 3 trial. Lancet Oncol 2019;20:924-37.

31 Paz-Ares L, Dvorkin M, Chen Y, et al. Durvalumab plus platinum-etoposide versus platinum-etoposide in first-line treatment of extensive-stage small-cell lung cancer (Caspian): a randomised, controlled, open-label, phase 3 trial. Lancet 2019;394:1929-39.

32 Hellmann MD, Ciuleanu T-E, Pluzanski A, et al. Nivolumab plus ipilimumab in lung cancer with a high tumor mutational burden. $N$ Engl J Med 2018;378:2093-104.

33 Borghaei H, Paz-Ares L, Horn L, et al. Nivolumab versus docetaxel in advanced Nonsquamous non-small-cell lung cancer. N Engl J Med 2015;373:1627-39.

34 Suzuki H, Hirashima T, Kobayashi M, et al. Carboplatin plus paclitaxel in combination with bevacizumab for the treatment of adenocarcinoma with interstitial lung diseases. Mol Clin Oncol 2013;1:480-2.

35 Shimizu R, Fujimoto D, Kato R, et al. The safety and efficacy of paclitaxel and carboplatin with or without bevacizumab for treating patients with advanced nonsquamous non-small cell lung cancer with interstitial lung disease. Cancer Chemother Pharmacol 2014;74:1159-66.

36 Brahmer JR, Lacchetti C, Schneider BJ, et al. Management of immune-related adverse events in patients treated with immune checkpoint inhibitor therapy: American Society of clinical oncology clinical practice guideline. JCO 2018;36:1714-68.

37 Naidoo J, Wang X, Woo KM, et al. Pneumonitis in patients treated with Anti-Programmed Death-1/Programmed death ligand 1 therapy. $J$ Clin Oncol 2017;35:709-17.

38 Su Q, Zhu EC, Wu J-B, et al. Risk of pneumonitis and pneumonia associated with immune checkpoint inhibitors for solid 
tumors: a systematic review and meta-analysis. Front Immunol 2019;10:108.

39 Zhang S, Liang F, Zhu J, et al. Risk of pneumonitis associated with programmed cell death 1 inhibitors in cancer patients: a metaanalysis. Mol Cancer Ther 2017;16:1588-95.

40 Chai Q-Q, Du J-Y, Zhu J, et al. The differences in the safety and tolerability of immune checkpoint inhibitors as treatment for nonsmall cell lung cancer and melanoma: network meta-analysis and systematic review. Front Pharmacol 2019;10:1260.

41 Huang Y-fang, Xie W-jie, Fan H-yu, et al. Comparative safety of PD-1/PD-L1 inhibitors for cancer patients: systematic review and network meta-analysis. Front Oncol 2019;9.

42 Huang Y, Fan $\mathrm{H}$, Li N, et al. Risk of immune-related pneumonitis for PD1/PD-L1 inhibitors: Systematic review and network meta-analysis. Cancer Med 2019;8:2664-74.

43 Magee DE, Hird AE, Klaassen Z, et al. Adverse event profile for immunotherapy agents compared with chemotherapy in solid organ tumors: a systematic review and meta-analysis of randomized clinical trials. Ann Oncol 2020;31:50-60.

44 Xu C, Chen Y-P, Du X-J, et al. Comparative safety of immune checkpoint inhibitors in cancer: systematic review and network metaanalysis. BMJ 2018;363:k4226.
45 Steele TA. Chemotherapy-Induced immunosuppression and reconstitution of immune function. Leuk Res 2002;26:411-4.

46 Krisl JC, Doan VP. Chemotherapy and transplantation: the role of immunosuppression in malignancy and a review of antineoplastic agents in solid organ transplant recipients. Am J Transplant 2017;17:1974-91.

47 Oppong E, Cato ACB. Effects of glucocorticoids in the immune system. Adv Exp Med Biol 2015;872:217-33.

48 Löwenberg M, Tuynman J, Bilderbeek J, et al. Rapid immunosuppressive effects of glucocorticoids mediated through Lck and Fyn. Blood 2005;106:1703-10.

49 Haanen JBAG, Carbonnel F, Robert C, et al. Management of toxicities from immunotherapy: ESMO clinical practice guidelines for diagnosis, treatment and follow-up. Annals of Oncology 2017;28:iv119-42.

50 Thompson JA. New NCCN guidelines: recognition and management of Immunotherapy-Related toxicity. J Natl Compr Canc Netw 2018;16:594-6.

51 Xiao Y, Yu S, Zhu B, et al. Rgmb is a novel binding partner for PD-L2 and its engagement with PD-L2 promotes respiratory tolerance. $J$ Exp Med 2014;211:943-59. 